\title{
Coniothyrium minitans, un agent de lutte biologique au service de la protection intégrée
}

\author{
Annette PENAUD ${ }^{1}$ \\ Hervé MICHI \\ ${ }^{1}$ CETIOM, Direction Technique - Pathologie \\ végétale, Campus de Grignon, Av. L. Brétignières, \\ FR - 78850 Thiverval-Grignon \\ $<$ penaud@cetiom.fr> \\ ${ }^{2}$ Belchim Crop Protection (France) S.A.
}

\begin{abstract}
Sclerotinia stem rot is a major disease on winter oilseed rape which causes severe yield losses twice a decade. Chemical control is usually applied at the beginning of flowering stage, every year. Because of a reduced use of chemicals expected, a biological control agent as Coniothyrium minitans could be usefull for controlling the disease. Experiments have mainly been carried out with Contans ${ }^{\circledR}$ WG, a ready-for-use formulation of $C$. minitans. Firstly effectiveness of $C$. minitans against sclerotia of Sclerotinia sclerotiorum was demonstrated. Then, its use on oilseed rape crops has been studied for several years in large size experimental plots. When it is incorporated into soil, C. minitans reduced the soil infestation by destroying sclerotia. By decreasing the soil infestation, $C$. minitans also reduced the primary inoculum pressure. Thus it allowed to reduce the sclerotinia risk and the disease incidence. The results are discussed according to the decision support system CETIOM in progress. Thus, a reduction of sclerotinia risk underneath a decision threshold in treated field plots could prevent any further spray of fungicides. However, it can be needed to apply fungicides which are more efficient when the disease pressure is reduced. These results contribute to integrate the use of Contans ${ }^{\circledR}$ WG in the disease management of sclerotinia stem rot of oilseed rape.
\end{abstract}

Key words: Contans ${ }^{\circledR}$ WG, winter oilseed rape, Sclerotinia sclerotiorum, Coniothyrium minitans, IPM (Integrated Protection Management)

Une autre voie est d'intégrer la lutte biologique dans les stratégies de protection fongicide du colza contre la sclérotiniose. Ceci est rendu possible grâce à la mise sur le marché d'une spécialité biologique Contans ${ }^{\circledR}$ WG, à base de spores du champignon Coniothyrium minitans spécifique des sclérotes de Sclerotinia sp.

\section{L'agent pathogène impliqué}

La sclérotiniose du colza est causée par Sclerotinia sclerotiorum. Ce champignon se conserve sur ou dans le sol sous forme de sclérotes dont la durée de vie est en moyenne de 3 à 5 ans, pouvant aller jusqu'à 10 ans selon le contexte pédoclimatique.

Parfois, en fin d'automne ou début d'hiver, il arrive que des sclérotes germent directement au contact des racines du colza et produisent un abondant mycélium blanc cotonneux (germination mycélienne) qui ceint le collet des plantes, envahit les feuilles de la rosette et par contact se propage de plante à plante lorsque celles-ci sont denses sur la ligne de semis. Si les plantes touchées sont entièrement détruites, elles ont auparavant permis au champignon de produire de très nombreux sclérotes qui renforcent le potentiel infectieux du sol. En général, c'est au printemps que les sclérotes superficiels ou enfouis dans les 2 à 3 premiers centimètres germent en produisant des apothécies beige clair, on parle alors de germination carpogénique. Produites au ras du sol, les apothécies libèrent, sous l'action de brusques variations $d$ 'hygrométrie, plusieurs milliers d'ascospores, qui entraînées au-dessus de la végétation, peuvent être transportées sur des distances de plusieurs dizaines de mètres. C'est ainsi qu'une parcelle indemne de sclérotes peut connaître une épidémie par le simple transport d'ascospores issues d'une parcelle infestée voisine. Dans ce cas, on note généralement un gradient d'importance décroissante de la bordure jouxtant la parcelle voisine contaminatrice vers l'intérieur de la culture.

Les ascospores disséminées vont alors polluer tous les organes végétatifs et floraux du colza mais seules les ascospores déposées sur les pétales sont capables de germer, de coloniser le pétale et de produire une masse mycélienne suffisante pour qu'après sa chute sur une feuille le mycélium soit en mesure de pénétrer dans les tissus foliaires.

Si les conditions climatiques sont favorables, le mycélium poursuit sa progression le long de la feuille pour atteindre la tige où il forme un manchon blanc caractéristique, centré au niveau $d^{\prime}$ une cicatrice foliaire et souvent accompagné de stries d'accroissement violacées. Cette pourriture entraîne une rupture de l'alimentation de 
la partie supérieure de la plante et son échaudage. Les siliques produites sont mal remplies de petites graines desséchées prématurément, ce qui entraîne des pertes de rendement de l'ordre de 1 à 1,5 q/ha par tranche de $10 \%$ de tiges attaquées. Dans et sur les tissus malades (tiges et siliques), le champignon forme en fin de cycle des sclérotes qui, à la faveur de la récolte et des façons culturales, tombent au sol où ils assurent la conservation du champignon pour un prochain cycle.

\section{Le mycoparasite des sclérotes : Coniothyrium minitans}

C. minitans est un mycoparasite qui cause la destruction des hyphes et des sclérotes de S. sclerotiorum.

Les hyphes de $C$. minitans pénètrent les sclérotes de $S$. sclerotiorum au travers de l'écorce pigmentée ou par des altérations à la surface du sclérote [1]. Puis ces hyphes se développent entre et dans les cellules internes du sclérote. Les hyphes prolifèrent à l'intérieur du sclérote et des pycnides sont formées à l'intérieur et sur le sclérote en moins de 15 jours en conditions optimales.

La pénétration et la dégradation des cellules interviennent par le biais de la production $d^{\prime}$ enzymes extracellulaires: chitinase et $\beta$ 1,3-glucanase. La $\beta$-1,3-glucanase semble jouer un rôle-clé dans le mycoparasitisme du C. minitans vis-à-vis de $\mathrm{S}$. sclerotiorum. Le gène cmg1 codant pour cette enzyme a été isolé [2] et de récents travaux suggèrent que l'activité de la $\beta$-1,3-glucanase serait corrélée au $\mathrm{pH}$ ambiant, dans une plage de 3 à 8 , avec un optimum pour un $\mathrm{pH}$ compris entre 4 et 6 . En revanche, un $\mathrm{pH}>7$ réduirait son activité [3]. La production d'enzymes extracellulaires ne semble pas être le seul mode d'action utilisé par C minitans pour le mycoparasitisme des sclérotes. II dégraderait aussi l'acide oxalique produit par S. sclerotiorum. Or, la dégradation de l'acide oxalique par $C$. minitans créerait des conditions de $\mathrm{pH}$ favorables à une bonne activité de l'enzyme $\beta$-1,3-glucanase.
C. minitans produirait aussi des substances antibiotiques et antifongiques telles que le macrosphelide A mise en évidence à partir de la souche anglaise CONIO [4], ce qui peut ne pas être le cas chez d'autres souches de C. minitans.

En effet, il existe une variabilité des souches de $C$. minitans, tant pour les caractéristiques morphologiques des colonies que pour leur capacité à produire des spores en quantité industrielle. C'est ainsi qu'au niveau international différentes équipes ont sélectionné leur souche. Parmi les plus citées en Angleterre IMI 134523 [5], aux Pays-Bas ITV1 [6], au Canada LRC 2137 [7], A69 en Nouvelle Zélande [8], en Chine ZS-1 ou Chy-1 [9] et en Allemagne CON/M/91-08 sélectionnée parmi 32 souches pour son agressivité et sa forte capacité de production de spores [10]. Un process de production sur milieu solide et une formulation innovante ont donné lieu à un produit homologué aujourd'hui dans de nombreux pays et commercialisé sous l'appellation de Contans ${ }^{\circledR}$ WG. En France, ce produit bénéficie depuis 2003 d'une AMM sans classement toxicologique pour l'usage Traitements généraux * traitement du sol * sclerotinia.

\section{Matériel et méthodes}

Depuis 2000, 3 types d'essais ont conduit à l'évaluation du C. minitans : i) directement sur les sclérotes qui constituent la cible du mycoparasite, ii) dans la durée d'une rotation et iii) en stratégies avec la lutte fongicide classique.

\section{Efficacité directe sur sclérotes}

Deux productions de C. minitans, respectivement la souche ITV1 et la souche CON/M/91-08 = Contans ${ }^{\circledR}$ WG, ont été comparées appliquées soit à l'automne, soit au printemps, à une dose équivalente à $4 \mathrm{~kg} / \mathrm{ha}$ de Contans ${ }^{\circledR}$ WG. La méthode consiste à pulvériser une suspension de spores de $C$. minitans sur 40 sclérotes disposés dans une terrine remplie de terre. Après application, les sclérotes sont recouverts d'une fine couche de terre avant d'être placés dans une culture de colza. Chaque modalité comporte 6 répétitions. Au printemps, les apothécies sont dénombrées pendant toute la période où elles sont formées par les sclérotes d'un témoin non traité.

\section{Efficacité dans la rotation}

Afin de préciser la persistance d'action du Contans ${ }^{\circledR}$, une série d'essais pluriannuels a été mise en place dans des rotations colza-céréales où, en détruisant progressivement le stock de sclérotes du sol, il est attendu une réduction de la pression parasitaire sur colza. Quatre dispositifs ont été conduits en Lorraine (54) et en Auvergne (03).

Pour s'affranchir des disséminations d'ascospores de S. sclerotiorum provenant du voisinage, les parcelles élémentaires sont de grande dimension, supérieures à $2500 \mathrm{~m}^{2}$ et le dispositif expérimental sans répétition est réduit à un couple de parcelles traitée et non traitée.

Les stratégies d'application de C. minitans sont résumées dans le tableau 1. Àl'automne 2000, le produit commercial Contans ${ }^{\circledR}$ WG a été appliqué à la dose $2 \mathrm{~kg} / \mathrm{ha}$, soit avant semis du colza dans deux des dispositifs, soit sur chaumes de colza juste avant le semis du blé dans les deux autres dispositifs. À l'automne 2001, les parcelles traitées l'année précédente ont reçu une application d'entretien de Contans ${ }^{\circledR}$ WG à une dose de $1 \mathrm{~kg} / \mathrm{ha}$ et dans l'un des dispositifs (Le Theil A), une nouvelle parcelle a été traitée à la dose de 2 kg/ha. À l'automne 2002, Contans $^{\circledR}$ WG a de nouveau été appliqué à $1 \mathrm{~kg} / \mathrm{ha}$ dans les parcelles déjà traitées les années précédentes, à l'exception de la parcelle du Theil B qui n'a reçu aucun traitement.

Chaque année, au moins 180 sclérotes, collectés dans des cultures infectées ou produits in vitro, sont placés dans des tamis, à raison de 10 sclérotes par tamis, et enfouis dans le sol des parcelles traitées ou non. À trois reprises au cours de la saison i) fin hiver, ii) début floraison et iii) à la récolte, soixante sclérotes sont exhumés et examinés pour apprécier visuellement leur état de dégradation. Cinq semaines avant récolte, l'effet des traitements avec C. minitans est apprécié sur colza par comptage de 4 fois 50 plantes prises au centre de chaque bande des dispositifs.

Tableau 1. Stratégies d'application de C. minitans dans une rotation colza-céréales.

\begin{tabular}{|lllll|}
\hline Lieux & & $\mathbf{2 0 0 0 - 0 1}$ & $\mathbf{2 0 0 1 - 0 2}$ & $\mathbf{2 0 0 2 - 0 3}$ \\
\hline Le Theil A (03) & Culture de la rotation: & Blé & Colza & Blé \\
& Dose de C. minitans & $2 \mathrm{~kg} / \mathrm{ha}$ & $1 \mathrm{~kg} / \mathrm{ha}$ & $1 \mathrm{~kg} / \mathrm{ha}$ \\
& Dose de C. minitans bis & & $2 \mathrm{~kg} / \mathrm{ha}$ & $1 \mathrm{~kg} / \mathrm{ha}$ \\
Le Theil B (03) & Culture de la rotation : & Colza & Blé & Triticale \\
& Dose de C. minitans & $2 \mathrm{~kg} / \mathrm{ha}$ & $1 \mathrm{~kg} / \mathrm{ha}$ & 0 \\
Juvrécourt (54) & Culture de la rotation : & Colza & Blé & Orge \\
& Dose de C. minitans & $2 \mathrm{~kg} / \mathrm{ha}$ & $1 \mathrm{~kg} / \mathrm{ha}$ & $1 \mathrm{~kg} / \mathrm{ha}$ \\
Gézoncourt (54) & Culture de la rotation : & Blé & Orge & Colza \\
& Dose de C. minitans & $2 \mathrm{~kg} / \mathrm{ha}$ & & $1 \mathrm{~kg} / \mathrm{ha}$ \\
\hline
\end{tabular}


Stratégies de protection contre la sclérotiniose à base de Contans ${ }^{\circledR}$ WG

Une expérimentation pluriannuelle de stratégies d'application de Contans ${ }^{\circledR}$ WG est menée par la firme Belchim depuis 2005 et réalisée au plein champ par les techniciens de la FREDON Bourgogne. Les essais sont réalisés en parcelles élémentaires d'au moins $400 \mathrm{~m}^{2}$.

Les stratégies se déclinent en parcelles traitées Contans ${ }^{\circledR}$ WG au semis du colza à la dose de $1 \mathrm{~kg} / \mathrm{h}$ a en 2005 ou à la dose de $2 \mathrm{~kg} / \mathrm{ha}$ à partir de 2006 et en parcelles non traitées. Une application fongicide ou non au printemps complète la stratégie d'automne. Deux familles de fongicides sont testés : les DMI avec l'emploi soit de metconazole soit de tébuconazole et un dicarboxamide avec boscalid (Pictor $\mathrm{Pro}^{\circledR}$ ) dont l'efficacité est reconnue supérieure à celle des DMI.

Pour mesurer l'effet du Contans ${ }^{\circledR}$ WG sur les sclérotes, une mise en place de sclérotes exogènes, récoltés l'année précédente dans des parcelles de colza contaminées par $S$. sclerotiorum, est réalisée au moyen de tamis enterrés dans I'horizon de sol traité. Repérés, ils sont ensuite observés 5 à 6 mois plus tard.

L'effet du Contans ${ }^{\circledR}$ WG sur le risque sclérotinia est estimé à l'aide d'un kit sclerotinia CETIOM. Pour réaliser ce kit au champ, 40 fleurs sont prélevées aléatoirement à la chute des premières pétales, au champ, puis disposées dans des boîtes de Petri renfermant un milieu semisélectif [11]. En présence de sclérotinia, ce milieu se décolore rapidement grâce à la présence d'un indicateur de $\mathrm{pH}$ dans le milieu qui vire du bleu au jaune dès lors que $S$. sclerotiorum en se développant produit suffisamment d'acide oxalique pour acidifier le milieu.

L'effet du traitement Contans ${ }^{\circledR}$ WG sur la culture est évalué par une observation 5 semaines avant récolte, effectuée sur 100 plantes par parcelle élémentaire afin d'estimer le pourcentage de tiges touchées par le sclérotinia. Par la suite, sur des parcelles présentant des niveaux d'attaque en sclérotinia supérieurs à $10 \%$, une récolte a été réalisée.

\section{Résultats}

\section{Efficacité directe de C. minitans sur sclérotes}

Les spores de C. minitans appliquées à l'automne sur des sclérotes placés dans des terrines sous un couvert de colza au champ (projet SPOREFUN) sont capables de réduire à néant la production d'apothécies au printemps suivant alors qu'elles n'empêchent pas l'apparition d'apothécies quand l'application est faite un mois avant la floraison (figure 1).
Après production des apothécies, pratiquement tous les sclérotes du témoin non traité sont retrouvés, avec $35 \%$ de sclérotes d'aspect sain et près de $60 \%$ de sclérotes naturellement altérés car épuisés par la production d'apothécies (figure 2). Dans les lots de sclérotes traités, il est à noter un effet très marqué de la date $\mathrm{d}^{\prime} \mathrm{appli-}$ cation du C. minitans sur l'état des sclérotes. Si I'application d'automne (T1 ou T2) laisse au plus $10 \%$ de sclérotes sains et entre 62 à $87 \%$ de sclérotes dans l'incapacité de produire des apothécies, l'application de printemps à l'apparition des stipes (T 3 ou T4) conduit à des proportions de sclérotes d'aspect sain et altérés équivalentes à celles du témoin non traité. Des deux préparations de C. minitans, c'est Contans ${ }^{\circledR}$ WG appliqué à l'automne (T2) qui enregistre la plus forte disparition de sclérotes avec près de $30 \%$ de sclérotes disparus et plus de $60 \%$ partiellement désintégrés. Ce résultat montre la nécessité d'un délai d'action suffisant pour une action optimale du mycoparasite.

\section{Évaluation du Contans ${ }^{\circledR}$ WG dans la durée d'une rotation}

En test d'efficacité directe, Contans ${ }^{\circledR}$ WG peut permettre de détruire $90 \%$ des sclérotes. Qu'en est-il dans les conditions de la pratique? Sachant que la survie du mycoparasite est de 12 à 18 mois sur sclérotes et qu'il se multiplie dans les sclérotes qu'il a parasités, C. minitans devrait être en mesure de se maintenir au cours du temps même s'il est redistribué dans le sol par les façons culturales (labour). Pour pallier sa dilution dans le sol, la stratégie poursuivie est $d^{\prime}$ 'entretenir la pression d'inoculum de l'agent biologique dans la rotation en administrant chaque année une dose d'entretien.

Au cours des trois campagnes d'étude, les attaques de sclérotinia dans les parcelles emblavées en colza ont été trop faibles, de l'ordre de 1 à $2 \%$, pour mettre en évidence des différences entre traitements. En revanche, des différences sont observées entre les sclérotes enfouis dans un sol traité ou non par C. minitans (tableau 2).

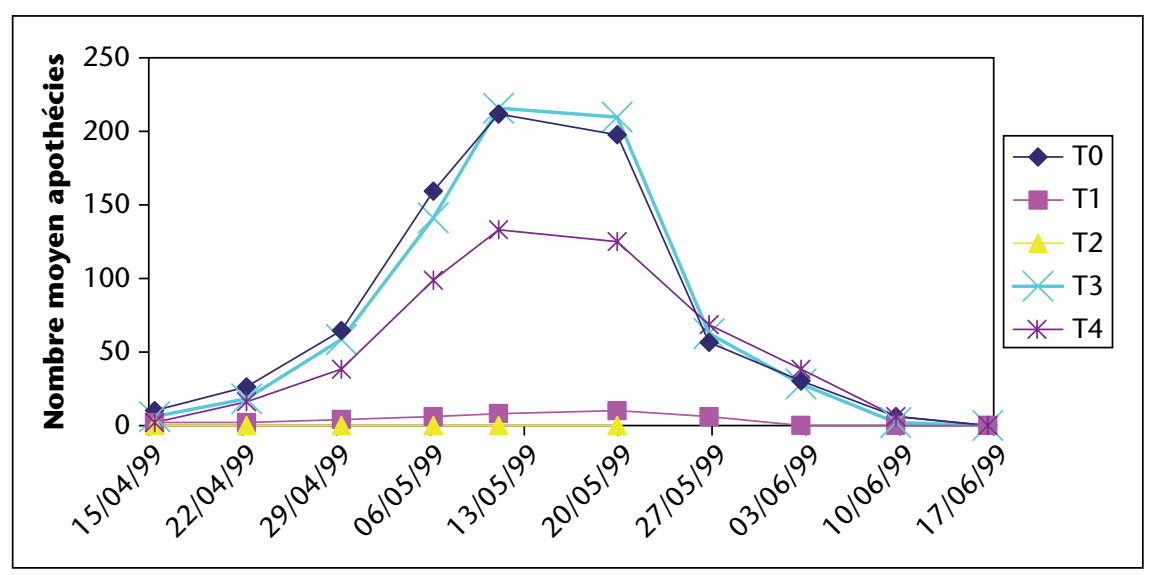

Figure 1. Nombre moyen d'apothécies en fonction des traitements appliqués. T0 = témoin non traité; $T 1$ = C. minitans ITV1 $10^{8}$ spores $/ \mathrm{m}^{2}$ appliqué le 25/11/98;T2 = C. minitans CON/M/91-08 $4 \mathrm{~kg} / \mathrm{ha}$ appliqué le $25 / 11 / 98 ; T 3=\mathrm{C}$. minitans ITV1 $10^{8}$ spores $/ \mathrm{m}^{2}$ appliqué le 23/03/99 sur premiers stipes; $T 4=\mathrm{C}$. minitans CON/M/91-08 $4 \mathrm{~kg} /$ ha appliqué le 23/03/99.

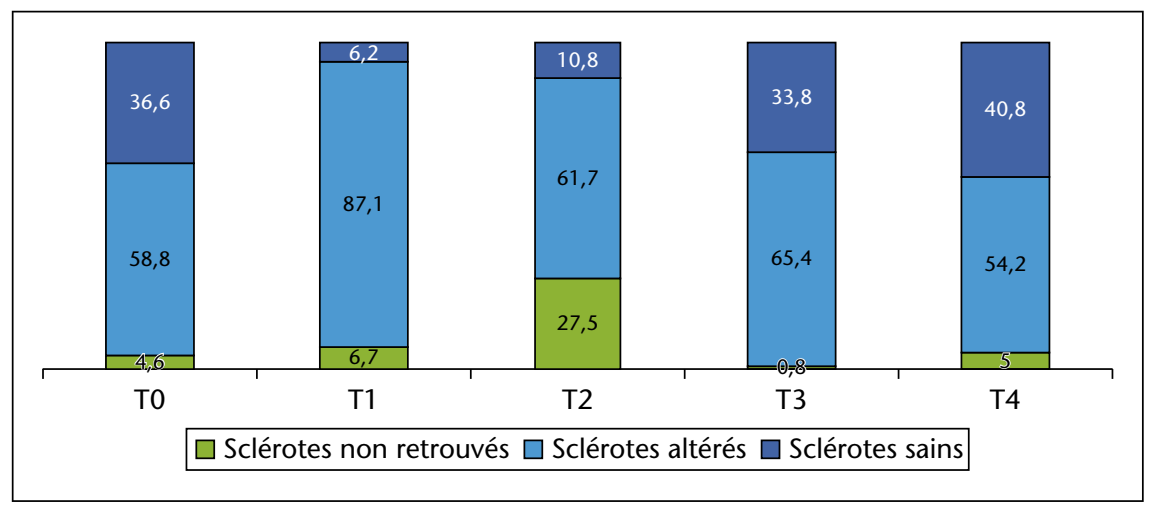

Figure 2. État des sclérotes après traitement et production d'apothécies (17/06/99). 
Tableau 2. Fréquence de sclérotes altérés-détruits en fonction des traitements appliqués dans rotation.

\begin{tabular}{|lllll|}
\hline Lieux & Traitements & \multicolumn{2}{c|}{$\begin{array}{c}\text { Pourcentage de sclérotes altérés ou détruits à } \\
\text { I'époque de la floraison du colza }\end{array}$} \\
\cline { 3 - 5 } & & $\mathbf{2 0 0 1}$ & $\mathbf{2 0 0 2}$ & $\mathbf{2 0 0 3}$ \\
\hline Le Theil A (03) & Non traité & 6.6 & 8.3 & 0 \\
& C. minitans & 23.3 & 15.4 & 22 \\
& C. minitans bis & - & 23.3 & 8.3 \\
Le Theil B (03) & Non traité & 20 & 5 & 13.6 \\
& C. minitans & 35 & 11.8 & 21.8 \\
Juvrécourt (54) & Non traité & 1.7 & 5 & 56 \\
& C. minitans & 16.7 & 4 & 88.5 \\
Gézoncourt (54) & Non traité & 11.7 & 0 & 62 \\
& C. minitans & 61.7 & 1.7 & 80 \\
\hline
\end{tabular}

Au moment de la floraison 2001, une proportion de sclérotes est naturellement altérée, variant de 2 à $12 \%$ selon les sites. Sur le site lorrain de Gézoncourt, C. minitans augmente jusqu'à 10 fois la destruction des sclérotes lorsque ces derniers ont été enfouis dans un sol traité à la dose de $2 \mathrm{~kg} / \mathrm{ha}$.

En 2002, les résultats diffèrent d'une région à l'autre. En Lorraine (54), malgré l'application d'une dose d'entretien de $1 \mathrm{~kg} / \mathrm{ha}$, peu de sclérotes sont altérés et/ou détruits à l'époque de la floraison du colza. L'origine in vitro des sclérotes, des températures subléthales au moment de I'application et des températures basses durant I'hiver pourraient expliquer ce manque d'efficacité. En Auvergne (03), sur le site du Theil-A, en plus d'une disparition naturelle de sclérotes de près de $10 \%$, deux à trois fois plus de sclérotes sont altérés après avoir été enfouis dans un sol traité respectivement à 1 et $2 \mathrm{~kg} / \mathrm{ha}$ de $\mathrm{C}$. minitans. Ces derniers résultats suggèrent un effet dose de l'agent biologique apporté sept mois plus tôt. Sous couvert de blé (Le Theil B), la tendance est la même que sous couvert de colza. Ainsi, quel que soit le couvert, dans les deux situations où le sol a été traité à la dose de $2 \mathrm{~kg} / \mathrm{ha}$ en 2001 puis 1 kg/ha en 2002, on observe l'année suivante deux fois plus de sclérotes altérésdétruits que dans les témoins non traités.

En 2003, des disparités régionales sont également observées. L'altération et la destruction naturelle des sclérotes sont au moins cinq fois plus importantes en Lorraine qu'en Auvergne. En présence d'un niveau d'altération et de destruction naturelle de l'ordre de $60 \%$ en Lorraine, $C$. minitans tend à causer une altération supplémentaire de sclérotes de $25 \%$ en moyenne. En Auvergne, sur le site du Theil-A, le cumul de trois traitements de $C$. minitans conduit à une altération de plus de $20 \%$ des sclérotes alors que deux traitements permettent seulement une réduction de la source $\mathrm{d}^{\prime}$ inoculum de $8 \%$. Parallèlement, sur le site du Theil- $B$, on note une légère tendance à davantage d'altération de sclérotes dans la parcelle traitée C. minitans les années précédentes. Dans ces deux sites, il semblerait que davan- au début de floraison du colza, au centre des parcelles expérimentales, à l'aide de l'indicateur de risque mis au point par le CETIOM. Pour l'ensemble des 9 sites $\times$ années, les parcelles traitées C. minitans accusent toutes une réduction du taux de fleurs contaminées. L'écart entre témoin et traité $C$. minitans variant de 5 à $45 \%$, l'écart moyen sur les 9 sites s'établit néanmoins à près de $20 \%$ (figure 3).

En considérant non plus la pression inoculum mais la pression maladie, l'incidence de la sclérotiniose dans les zones témoins atteint au moins le seuil de nuisibilité de $10 \%$ et dépassent même les $75 \%$ de pieds touchés en 2007, année particulièrement favorable à l'expression de la maladie. Dans la zone traitée C. minitans, les taux d'attaque se révèlent significativement inférieurs à ceux de la zone non traitée. De plus, dans 3 des sites suivis, un effet dose d'application du C. minitans est observé, la dose de $2 \mathrm{~kg} / \mathrm{ha}$ conduisant à une réduction des attaques supérieure à celle observée pour la dose de $1 \mathrm{~kg} / \mathrm{ha}$ (figure 4).

\section{Stratégies de protection à base de Contans ${ }^{\circledR}$}

Alors que C. minitans a été incorporé au semis, la pression d'inoculum sclérotinia est mesurée tage de sclérotes soient altérés ou détruits après avoir été traités deux années consécutives à une dose de $2 \mathrm{~kg} / \mathrm{ha}$.

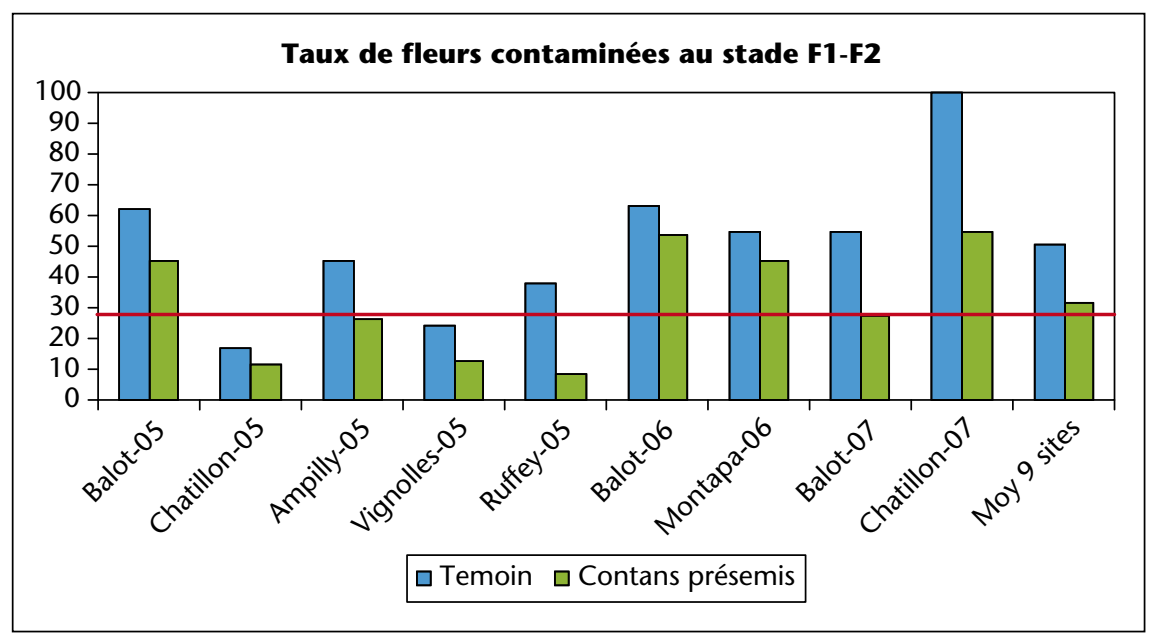

Figure 3. Taux de fleurs contaminées en zone témoin et en zone ayant reçu une application de Contans ${ }^{\circledR}$ WG à la dose de $1 \mathrm{~kg} / \mathrm{ha} \mathrm{(2005)} \mathrm{ou} 2 \mathrm{~kg} / \mathrm{ha}$ (2006-07).

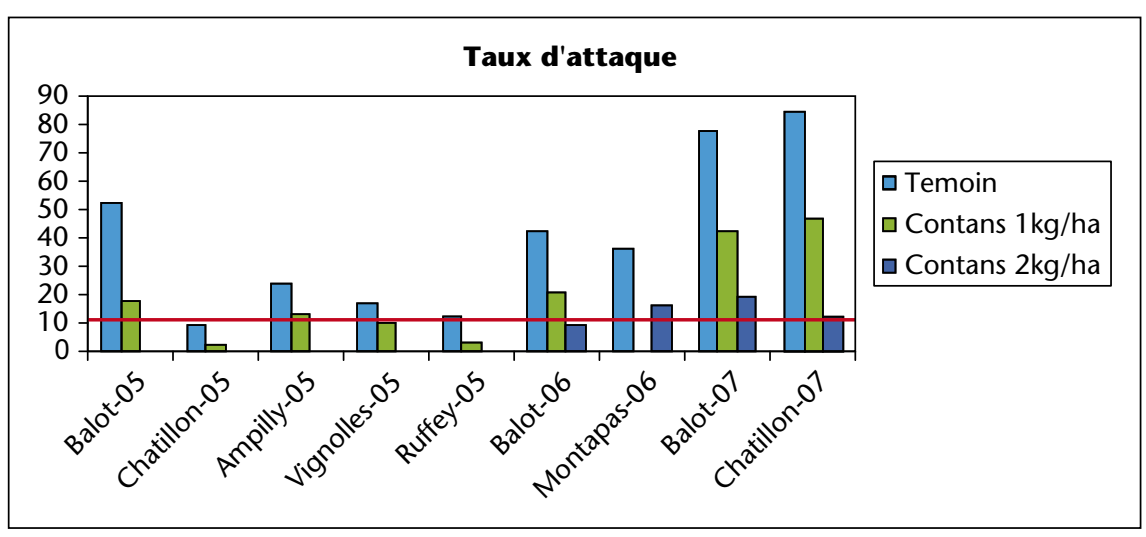

Figure 4. Niveau de l'attaque de sclérotonia (en fréquence de pieds touchés) en zone témoin et en zone traitée Contans ${ }^{\circledR}$ WG sur les 9 parcelles suivies entre 2005 et 2007. 
Une intégration des résultats dans le système $\mathrm{d}$ 'aide à la décision de gestion du risque sclerotinia à la parcelle travaillé au CETIOM depuis 2002 est obtenue en projetant respectivement pour les zones témoins et les zones traitées $C$. minitans l'incidence de la maladie en fonction de l'indicateur de risque PFI. II apparaît ainsi que le traitement $C$. minitans contribue globalement à abaisser le niveau de risque sclérotinia dans 8 situations sur 9 (figure 5).

Un seuil de décision de l'indicateur de risque sclérotinia a été établi à $30 \%$ de sorte que le risque pris de ne pas traiter alors que l'attaque finale serait supérieure au seuil de nuisibilité soit en moyenne de $10 \%$. En considérant ce seuil de décision $\mathrm{PFI}<30 \%$, l'outil détecte 3 situations traitées C. minitans où l'application complémentaire d'un traitement fongicide pouvait être évitée, les attaques en culture n'excédant pas $10 \%$. Pour un seuil de décision PFI > $30 \%$, l'outil décèle également 3 situations traitées C. minitans (C7, M6 et B5) où un traitement fongicide en végétation se justifiait mais où toutefois les attaques sont significativement réduites et ne dépassent pas $20 \%$ de plantes touchées comparées aux attaques des parcelles non traitées comprises entre $35 \%$ et $85 \%$. Par contre, l'outil est sévèrement mis en défaut dans une situation (B7) où avec un PFI avoisinant les $30 \%$ le taux de plantes attaquées atteint $50 \%$. Par ailleurs, le regroupement de 4 essais de stratégies d'application Contans ${ }^{\circledR}$ WG complétées ou non d'un traitement fongicide au printemps offre des tendances intéressantes (figure 6). Dans un contexte de fortes attaques de sclérotinia avec une attaque moyenne des 4 témoins à $51 \%$, la seule application de Contans ${ }^{\circledR}$ $2 \mathrm{~kg} / \mathrm{ha}$ est en tendance aussi efficace qu'un traitement fongicide à base de DMI appliqué à la chute des premiers pétales du colza. Si l'efficacité de la stratégie Contans ${ }^{\circledR} 2 \mathrm{~kg} /$ ha puis DMI n'est pas l'addition des efficacités individuelles, la tendance donne tout de même un gain d'efficacité de $20 \%$ par rapport au seul traitement fongicide. Même avec un fongicide plus efficace tel que Pictor Pro ${ }^{\circledR}$ (boscalid), la tendance reste, avec cependant un gain d'efficacité plus modeste de $10 \%$.

Concernant les rendements associés à ces différentes stratégies, les traitements uniques de Contans ${ }^{\circledR}$ WG ou de fongicides conduisent à des gains de rendement de 6 à 10 q/ha comparés aux témoins non traités (figure 7). Tout comme pour les efficacités, les gains de rendements les plus élevés sont obtenus à la faveur des stratégies Contans ${ }^{\circledR}$ WG présemis puis fongicide au stade G1 du colza.

\section{Discussion}

Le principal mode d'action de C. minitans est de parasiter les sclérotes de S. sclerotiorum

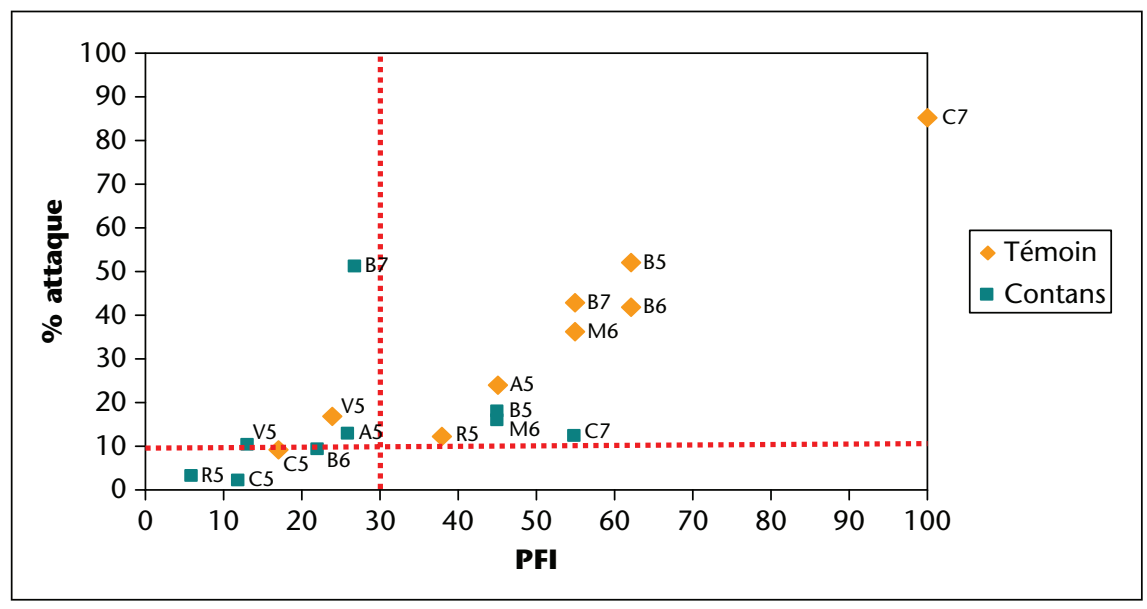

Figure 5. Projection des résultats Contans ${ }^{\circledR}$ WG dans I'outil d'aide à la décision de gestion du risque Sclérotinia à la parcelle.

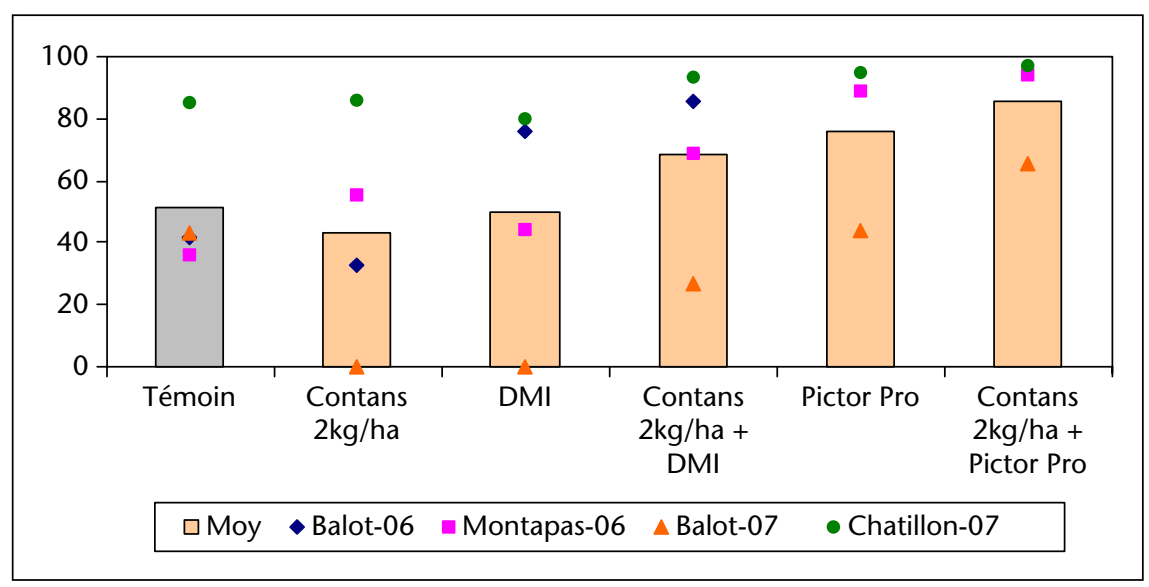

Figure 6. Efficacité des stratégies à base de Contans ${ }^{\circledR} 2 \mathrm{~kg} / \mathrm{ha}$.

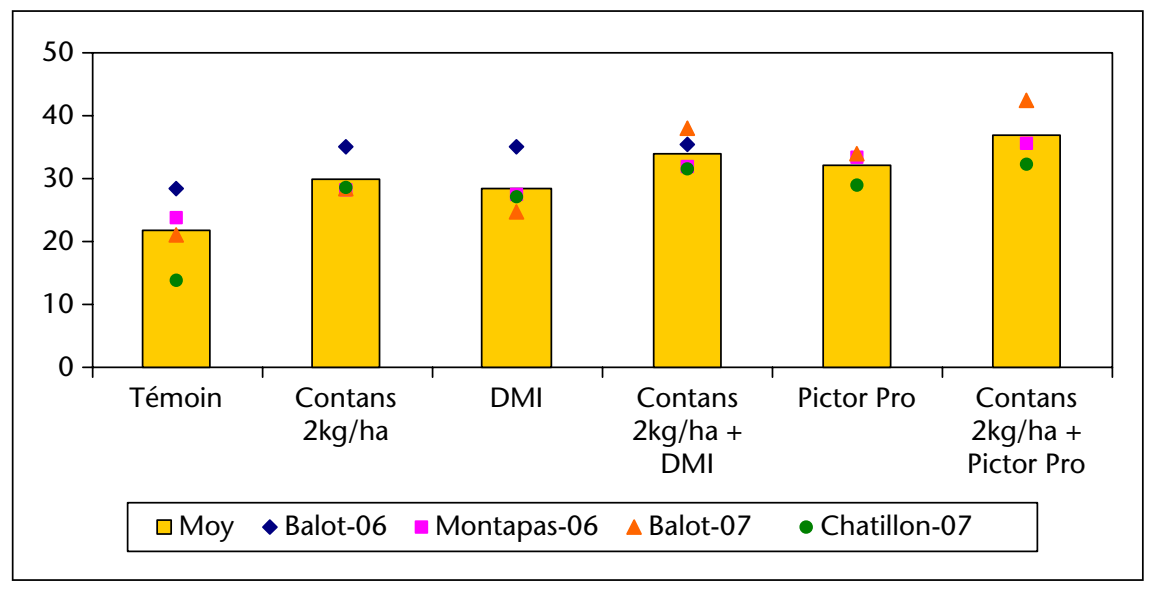

Figure 7. Rendements moyens en fonction des stratégies à base de Contans ${ }^{\circledR} 2 \mathrm{~kg} / \mathrm{ha}$ poursuivies. 
[12]. Sa mise en contact direct avec des sclérotes sous forme d'une pulvérisation d'une préparation de spores conduit ce mycoparasite à détruire ou à rendre inopérants en moyenne $40 \%$ des sclérotes traités comme le montrent nos résultats expérimentaux [13].

Lorsque les sclérotes sont parasités, ils se révèlent hors d'état de produire des apothécies. Ainsi la production d'ascospores à l'origine des contaminations du colza s'en trouve réduite comme cela est mis en évidence grâce à I'utilisation du kit de diagnostic permettant d'estimer la proportion de fleurs contaminées en tout début floraison du colza [14]. De plus, il apparaît que la pression d'inoculum est $\mathrm{d}^{\prime}$ autant plus faible que la dose de $C$. minitans est plus concentrée. Ce kit se révèle être un outil d'autant plus intéressant pour évaluer l'efficacité du C. minitans que suite à des conditions climatiques défavorables à l'expression de la maladie, les attaques en culture sont très souvent quasi inexistantes.

Néanmoins, le niveau d'attaque de la culture reste le paramètre d'évaluation de l'efficacité du C. minitans le plus pertinent. Dans les essais de Bourgogne, une application de C. minitans (2 kg/ha de Contans ${ }^{\circledR}$ WG) au semis permet une réduction significative des attaques. Ces résultats corroborent l'expérience allemande qui fait état de réductions d'attaque de l'ordre 20 à $30 \%$ en grandes parcelles de colza [10].

Par ailleurs, I'utilisation de C. minitans est à inscrire dans la durée car d'une part l'expérience montre que tous les sclérotes ne sont pas détruits à $100 \%$ après une seule application et d'autre part que l'efficacité tend à augmenter par des applications répétées sur la culture sensible et mieux encore sur l'ensemble des cultures de la rotation [15]

Par ailleurs, si I'utilisation de $C$. minitans sur une base de $2 \mathrm{~kg} / \mathrm{ha}$ de Contans ${ }^{\circledR} \mathrm{WG}$ en présemis incorporé peut constituer à elle seule une solution alternative à la protection fongicide de printemps, elle gagne cependant à être inté- grée dans une stratégie de complémentarité des luttes biologique et chimique, surtout en situation à risque sclerotinia élevé. Encore faut-il pouvoir être en mesure d'évaluer le risque sclérotinia. Le recours à un outil d'aide à la décision, qui permette sans trop de risque de décider de la nécessité ou non d'un traitement fongicide, offre la possibilité de raisonner l'intervention fongicide qui ne sera appliquée que si elle se justifie. Dans ce cas, notre agent biologique C. minitans constitue non seulement un outil alternatif de lutte mais se pose aussi comme un réel outil de protection intégrée.

\section{RÉFÉRENCES}

1. Huang HC, Kokko EG. Ultrastructure of hyperparasitism of Coniothyrium minitans on sclerotia of Sclerotinia sclerotiorum. Can J Plant Pathol 1987 ; 65 : 2483-9.

2. Giczey G, Kerenyi Z, Fülöp L, Hornok L. Expression of cmg1, an exo- $\beta$-1,3-glucanase gene from Coniothyrium minitans, increases during sclerotial parasitism. Appl Environ Microbiol $2001: 865-71$.

3. Li R, Li G, Han YC, Jiang DH, Huang H. Degradation of acid oxalic by Coniothyrium minitans and its effects on production and activity of this mycoparasite. Biol Control $2007 ; 43$ : 1-11.

4. McQuilken M, Gemmell J, Hill R, Whipps J. Production of macrosphelide A by the mycoparasite Coniothyrium minitans. FEMS Microbiology Letters $2003 ; 219: 27-31$.

5. Turner \& Tribe. On Coniothyrium minitans and its parasitism of Sclerotinia species. Trans $\mathrm{Br}$ Mycol Soc 1976 ; 66 : 97-105.

6. De Vrije T, Antoine N, Buitelaar R, Bruckner S, Dissevelt $M$, Durand $A$, et al. The fungal biocontrol agent Coniothyrium minitans: production by solid-state fermentation, application and marketing. App Microbiol Biotechnol 2001 ; $56: 58-68$.
7. Huang HC, Scott Erickson R. Factors affecting biological control of Sclerotinia sclerotiorum by fungal antagonists. J Phytopathology 2008; $156: 628-34$.

8. Ridgway, et al. Molecular marker assisted detection of the mycoparasite Coniothyrium minitans A69 in soil. New Zealand Protection $2000 ; 53: 114-5$.

9. Yang R, et al. Supression of Sclerotinia sclerotiorum by antifungal substances produced bu the mycoparasite Coniothyrium minitans. Eur J Plant Pathol $2007 ; 119$ : 411-20.

10. Lüth P. Biological control of Sclerotinia sclerotiorum in oilseed rape and sunflower with the fungal antagonist Coniothyrium minitans and improvement of its use. IOBC Bulletin 1995 ; $18: 18-25$.

11. Steadman JR, Marcinkowska I, Rutledge S. A semi selective medium for isolation of Sclerotinia sclerotiorum. Can / Plant Pathol $1994 ; 10$ : 159-65.

12. Whipps JM, Gerlagh M. Biology of Coniothyrium minitans and its potential for use in disease biocontrol. Mycological research 1992; 96 : 897-907.

13. Cael N, Penaud A. Des éléments nouveaux dans la lutte contre le sclerotinia du colza et du tournesol: un nouveau fongicide biologique à base d'un champignon antagoniste Coniothyrium minitans. In : $2^{e}$ Conférence Internationale sur les moyens alternatifs de lutte contre les organismes nuisibles aux végétaux. Lille 4, 5, 6 et 7 mars 2002.

14. Uzel et al. Contans, un outil complémentaire dans la protection du colza contre le sclérotinia. In : $3^{e}$ Conférence Internationale sur les moyens alternatifs de lutte contre les organismes nuisibles aux végétaux. Lille 13, 14 et 15 mars 2006.

15. Penaud $A$, Chollet $D$, Jung $L$, Biological control of Sclerotinia sclerotiorum by Coniothyrium minitans in crops rotations including OSR in France. In: 11th Int. Rapeseed Congr. Cohenhagen, July 2003. 